\title{
Surface True and Leaky Waves in Subsonic and Supersonic Regions. Explicit Solution of a Simple Model
}

\author{
D. TRZUPEK ${ }^{a, b, *}$ AND P. ZielińsKi ${ }^{a, c}$ \\ ${ }^{a}$ The H. Niewodniczański Institute of Nuclear Physics PAN, Radzikowskiego 152, 31-342 Cracow, Poland \\ ${ }^{b}$ Institute of Physics, Jagiellonian University, Reymonta 4, 30-059 Cracow, Poland \\ ${ }^{c}$ Institute of Physics, Cracow University of Technology, Podchorążych 1, 30-084 Cracow, Poland \\ A simple model of a surface of a $2 \mathrm{D}$ continuum is shown to exhibit subsonic and supersonic surface resonances \\ (leaky waves, pseudosurface waves), i.e. finite-lived excitations, along with usual surface waves (true subsonic \\ surface waves). The physical meaning, controversial in the existing literature, of the surface leaky waves in both \\ regions is discussed by a thought experiment in which a stimulus adjusted to the resonance parameters is applied \\ to the surface. The infinite displacement-stimulus ratio in the long-time limit then is shown to be in power in \\ all the cases of surface states and a finite amplitude of the leaky waves is demonstrated despite an apparent \\ divergence suggested by their algebraic form.
}

PACS numbers: 63.20.D-, 68.35.Ja

\section{Introduction}

The presence of surfaces breaks the translational invariance of the material and, therefore, gives rise to new vibrational states showing amplitudes that decrease with the depth into the bulk. The kind of motion would be precluded if the material had no bounds because the amplitude then would increase infinitely on the side of vacuum. When the system remains spatially periodic in the surface plane the wave vectors parallel to the surface are "good quantum numbers" so that the localized vibrations propagate along the surface as surface waves. The phenomenon occurs in a large range of scales starting from surfaces of stars and of the Earth, to those of crystals and nanoparticles.

Acoustic surface waves have been described for the first time by Lord Rayleigh in 1887. Their frequencies are always lower than those of all the bulk waves with the same component of the wave vector parallel to the surface. Modifications of surfaces by some adlayers or, on the atomic scale, adatoms, may however, bring the frequencies of surface vibrations to the regions where the propagation of bulk waves conveys the energy to the bulk. Then the surface wave acquires a finite lifetime and becomes a surface resonance. In the next section we give the rudiments of the mathematical treatment of the surface waves. In Sect. 3 we construct a simple model showing both, surface waves and surface resonances. The model allows one to obtain all the characteristics of the

* corresponding author; e-mail: Dominik.Trzupek@ifj.edu.pl vibrational surface states in an analytical way. We then discuss, in Sects. 4 and 5, the physical interpretation of apparently paradoxical solutions of the governing equations exhibiting an increase in amplitude in the direction into the bulk.

\section{Basic definitions}

The dynamics of a system consisting of a semi-infinite substrate terminated by a surface is governed by the equations of motion of the bulk material and by the boundary conditions imposed by the properties of the surface.

\subsection{Bulk radiative bands}

The solutions of the governing equations in the bulk region are, in the harmonic approximation, combinations of the Bloch waves

$$
w_{\mu}(\boldsymbol{r}, t)=e_{\mu}(\boldsymbol{k}, \omega) \mathrm{e}^{-\mathrm{i} \omega t+\mathrm{i} \boldsymbol{k} \cdot \boldsymbol{r}},
$$

where $\boldsymbol{k}$ is a wave vector from the first Brillouin zone, $\omega$ is the corresponding frequency and $e_{\mu}, \mu=1,2, \ldots, n$ is the polarization vector. The dimension $n$ of the polarization vector equals the number of the degrees of freedom of the unit cell of the material. The polarization vectors are the eigenvectors and the frequencies $\omega_{j}^{2}(\boldsymbol{k})$, $j=1,2, \ldots, n$ are the eigenvalues of a $\boldsymbol{k}$-dependent $n \times n$ matrix $M(\boldsymbol{k}, \omega)$. The secular equation $\operatorname{det} M\left(\boldsymbol{k}, \omega_{j}\right)=0$ is, thus, fulfilled defining the dispersion relations $\omega_{j}(\boldsymbol{k})$ of the bulk waves. Decomposing the wave vector into the components $k_{\|}$and $k_{\perp}$, parallel and perpendicular to the surface, respectively, one can solve the secular equation $\operatorname{det} M\left(k_{\|}, k_{\perp}, \omega\right)=0$ for the unknown $k_{\perp}$ at any point of 
the plane $\left(k_{\|}, \omega\right)$. The regions in this plane where at least one of the resulting $k_{\perp}$ is purely real support bulk homogeneous waves. These are, thus, radiative regions also called bands of the bulk waves or, simply, bulk bands. The bulk bands can be also viewed as the projection of the bulk dispersion relations onto the plane $\left(k_{\|}, \omega\right)$. The system of the bulk bands depends on the spatial orientation of the surface (Miller indices in the case of flat surfaces) but are independent of the properties of the surface itself, i.e. of the boundary conditions of the dynamical problem.

Complex solutions $k_{\perp}$ of the secular equation at real frequency $\omega$ with $\operatorname{Im} k_{\perp}>0$ describe near fields, also called evanescent partial waves, that decay exponentially with the distance from the surface into the bulk. Such solutions are rejected in the infinite system as they would diverge on the vacuum side of the surface. They are, however, finite everywhere at the presence of the surface. In summary, any solution of the dynamical problem of a medium terminated by a surface in the harmonic approximation is a linear combination of the partial homogeneous and/or evanescent bulk waves.

\subsection{Surface vibrational states}

The equations of motion of the surface layer constitute the boundary conditions of the problem. The solution of the whole dynamical problem for given $k_{\|}$and $\omega$, with or without an external perturbation applied to the surface, then is such a combination of bulk and evanescent partial waves that satisfies the set of boundary conditions. The solutions coming about for real frequencies with zero external perturbation constitute the surface states or, equivalently, surface waves. If they occur outside the bulk bands, i.e., by definition, have all the partial waves decreasing into the bulk, they are then called true surface waves, as they are really confined to the surface region. Whenever such waves persist down to the long wavelength limit, their phase speeds of propagation along the surface are necessarily lower than the sound speed of the slowest acoustic bulk wave in the same direction. Therefore, they are called subsonic surface waves. The existence of supersonic surface waves, i.e. the ones occurring in the long wavelength limit for real frequencies within radiative bands has been shown possible at certain orientations of surfaces in some symmetries of the bulk materials. Their dispersion relations, represented, in this limit, by single straight lines on the background of the radiative bands earned them the name "secluded surface waves" [1]. Every [2] has found analogous waves on an interface consisting of coplanar thin cavities in a continuous medium. Recently the present authors have shown a possibility of existence of true surface waves occurring at one single point of a radiative band in materials possessing rotational degrees of freedom of rigid constituents [3] and for a continuum covered by a specific surface [4]. Such waves have been called isolated true surface waves [5].

Apart from the solutions with real frequencies the boundary conditions may yield solutions with complex frequencies. The condition $\operatorname{Im} \omega<0$ then ensures an exponential time decay of the corresponding excitations with the time constant $\tau=1 / \operatorname{Im} \omega$. In the long wavelength and low frequency limit or, equivalently, in a continuum with no internal degrees of freedom, the corresponding wave vector $k_{\perp}$ must have also $\operatorname{Im} k_{\perp}<0$, i.e. the corresponding wave increases in amplitude in the direction into the bulk. The mechanism underlying the finite lifetime is simple: a part of vibrational energy is carried away into the bulk at every swing by the travelling bulk waves like a liquid from a leaking pot. Therefore, the corresponding finite-life-time excitations are called leaky waves [6]. Sometimes the names "pseudosurface wave" or "surface resonance", in analogy with impurity resonance, are also used. Once the real part of the frequency Re $\omega$ of such a wave lies in the bulk radiative band, the local density of surface states shows a peak of width defined by $\operatorname{Im} \omega$. Then one speaks of supersonic surface leaky wave.

However, leaky waves with $\operatorname{Re} \omega$ lying outside the bulk bands are also found in some specific systems, such as high index surfaces of crystals and solid-liquid interface [7]. The physical significance of such solutions as well as the interpretation of the spatial infinite increase in amplitude with the distance from the surface implied by the leaky waves remains controversial. In the next section we propose a simple model in which both surface subsonic and supersonic waves may exist as well as ordinary true subsonic surface wave. A simple thought experiment will then explain the physical meaning of the surface states found.

\section{A simple model showing subsonic and supersonic leaky waves}

A 2D planar isotropic continuum terminated by a straight edge (surface) of linear density $\rho_{\mathrm{s}}$ placed in a local harmonic potential is probably the simplest model in which the analytical solutions can be obtained for the true subsonic surface waves as well as for the supersonic and subsonic leaky waves. The equation of motion for the displacement $u(x, y, t)$ perpendicular to the plane of the system in the bulk reads

$$
\frac{\partial^{2} u}{\partial t^{2}}=c^{2}\left(\frac{\partial^{2} u}{\partial x^{2}}+\frac{\partial^{2} u}{\partial y^{2}}\right),
$$

where $c$ is the phase velocity. For the sake of simplicity we put the $x$ axis along the edge of the system. Any solution in the bulk then has a generalized d'Alembert form

$$
u(x, y, t)=u(\zeta),
$$

where

$$
\zeta=\hat{k}_{\|} x+\hat{k}_{\perp} y-c t .
$$

The components $\hat{k}_{\|}=k_{\|} / \sqrt{k_{\|}^{2}+k_{\perp}^{2}}$ and $\hat{k}_{\perp}=$ $k_{\perp} / \sqrt{k_{\|}^{2}+k_{\perp}^{2}}$ form a unit wave vector with, however, a norm being a sum of squares and not a sum of squared moduli of the wave vector components. A Bloch wave (Eq. (1)) is of course a particular case of the solution 
given by Eq. (3) with the dispersion relation

$$
\omega^{2}=c^{2}\left(k_{\|}^{2}+k_{\perp}^{2}\right) .
$$

Equation (3) allows one to relate the time derivative of the displacement $u$ with its gradient being in this case identical with the directional derivative in the direction perpendicular to the wave front, so that $\operatorname{grad} u=-\frac{1}{c} \frac{\partial u}{\partial t}$. Consequently, in particular

$$
\frac{\partial u}{\partial y}=\hat{k}_{\perp} \operatorname{grad} u=-\frac{\hat{k}_{\perp}}{c} \frac{\partial u}{\partial t} .
$$

The boundary condition at $y=0$ is given by the equation of motion of the linear density $\rho_{\mathrm{s}}$ placed in a harmonic potential $V=\frac{1}{2} K u$. This 1D surface is coupled with the substrate by elastic forces with the stiffness constant $T$ and subjected to an external oscillating force of the amplitude $f_{0}$ :

$$
\rho_{\mathrm{s}} \frac{\partial^{2} u}{\partial t^{2}}=\left.T \frac{\partial u}{\partial y}\right|_{y=0}-K u+f_{0} \mathrm{e}^{\mathrm{i} k_{\|} x-\mathrm{i} \omega t} .
$$

Inserting the relation (6) into Eq. (7) one obtains an effective ordinary equation of motion of a forced, damped oscillator

$$
\rho_{\mathrm{s}} \frac{\mathrm{d}^{2} u}{\mathrm{~d} t^{2}}+\frac{T k_{\perp}}{c} \frac{\mathrm{d} u}{\mathrm{~d} t}+K u=f_{0} \mathrm{e}^{\mathrm{i} k_{\|} x-\mathrm{i} \omega t} .
$$

The reduction of the boundary condition to an ordinary differential equation is possible when the dispersion relation in the substrate is represented by a straight line at any direction in the reciprocal space, see Eq. (5). In general, however, an integro-differential equation has to be solved due to the dispersion of the phase velocity [8].

\section{True and leaky surface waves: dispersion relations}

The bulk band in the present model is represented in Fig. 1 by a shaded area. It is limited from below by the straight line $\omega=c k_{\|}$. The solutions of the bulk equation of motion (2) with the boundary condition ( 7 ) for $f_{0}=0$ correspond to the surface states. Their general form is $u(x, y, t)=u_{0} \mathrm{e}^{\mathrm{i} k_{\|} x+\mathrm{i} k_{\perp} y-\mathrm{i} \omega t}$. The loci of the real parts of their frequencies are shown in Fig. 1 using the following reduced quantities:

$$
\begin{aligned}
& \tilde{K}=\frac{K \rho_{\mathrm{s}} c^{2}}{T^{2}}, \quad \tilde{k}_{\|}=\frac{k_{\|} \rho_{\mathrm{s}} c^{2}}{T}, \quad \tilde{k}_{\perp}=\frac{k_{\perp} \rho_{\mathrm{s}} c^{2}}{T}, \\
& \tilde{\omega}=\frac{\omega \rho_{\mathrm{s}} c}{T} .
\end{aligned}
$$

The frequencies are expressed explicitly by the formula

$$
\tilde{\omega}^{2}=\tilde{K}-\frac{1}{2} \pm \sqrt{\tilde{k}_{\|}^{2}-\tilde{K}+\frac{1}{4}} .
$$

The physically meaningful frequencies have $\operatorname{Im} \omega \leq 0$. In the solution represented by the line $A$ the frequency is real, i.e. $\operatorname{Im} \omega=0$ and the wave vector $k_{\perp}$ is purely imaginary with $\operatorname{Im} k_{\perp}>0$. This is, therefore, a subsonic true surface wave, decreasing exponentially with the depth into the bulk. Its dispersion relation merges with the bulk band, so that it should not be called "acoustic". The portion $B$ of the line shown in Fig. 1 is char-

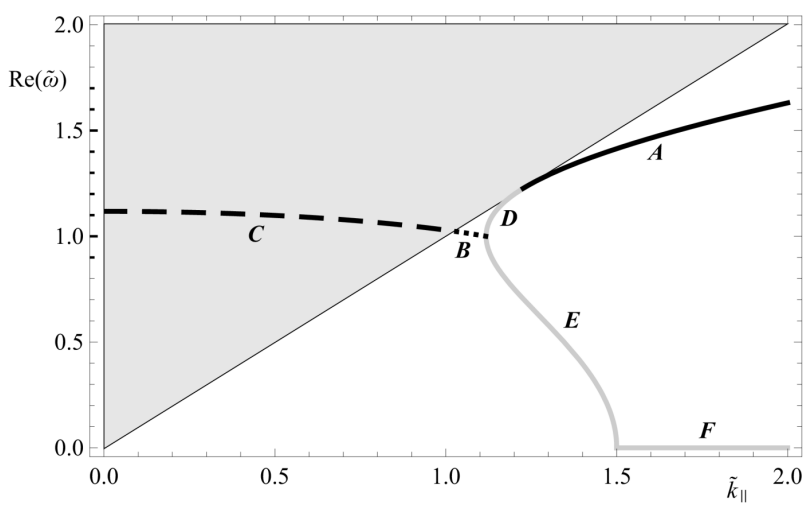

Fig. 1. Bulk band (shaded) and dispersion relation of surface states in model of $2 \mathrm{D}$ continuum terminated by 1D surface. True subsonic surface wave $(A)$, subsonic leaky wave $(B)$, supersonic leaky $(C)$. Segments $D$ and $E$ showing real frequency and $\operatorname{Im} k_{\perp}<0$ have no physical manifestation.

acterized by a complex frequency $\operatorname{Im} \omega<0$. As can be seen from Eq. (5), the complex wave vector $k_{\perp}$ must then have a negative imaginary part $\operatorname{Im} k_{\perp}<0$ implying an increase in the amplitude in the direction into the bulk. This is, therefore, a subsonic leaky wave. The continuation of this curve inside the bulk band, shown in Fig. 1 as the line $C$, has analogous characteristic except that the real part of the frequency lies in the bulk band, i.e. $\operatorname{Re} \omega>c k_{\|}$.

This is typical of supersonic surface leaky wave. The portions $D$ and $E$ of the line seen in Fig. 1 turn out to show real frequency and $\operatorname{Im} k_{\perp}<0$. Such solutions seem to have no physical meaning. This supposition is supported by the calculus of the local density of states obtained with a weak additional damping. The latter may be realized as putting the system into a viscous liquid. The result is depicted in Fig. 2. The true subsonic surface wave is represented by a narrow ridge visible in the upper right part of the plot. The supersonic leaky wave is also discernible as a broad maximum within the bulk band on the left hand side. The subsonic leaky wave has no direct manifestation in this plot. In fact, it results in an extra density of states represented by a large maximum in the center of the plot just above the lower limit of the bulk band. Really, the extra states in this region are responsible for the radiation of energy ("leakage") related to this subsonic leaky wave. The portions $D$ and $E$ of the curve of Fig. 1 leave no traces in the local density of states that confirms their lack of physical meaning.

\section{True and leaky surface waves: temporal behaviour}

The reaction of the system to the oscillating external perturbation $f_{0} \mathrm{e}^{\mathrm{i} k_{\|} x-\mathrm{i} \omega t}$ with the frequency turning the left hand side of Eq. (8) into zero can be studied by the method of variation of parameters. In particular, we look for the solution of Eq. (8) in the form 


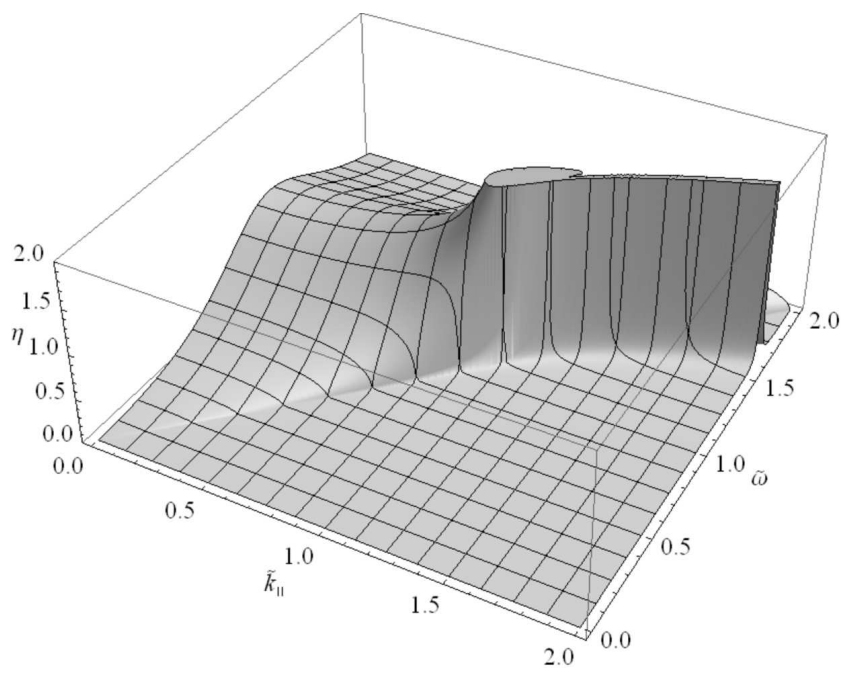

Fig. 2. Local density of states in system of Fig. 1.

$u(x, y=0, t)=A(t) \mathrm{e}^{\mathrm{i} k_{\|} x-\mathrm{i} \omega t}$ and insert it into Eq. (8). A simple calculus shows that the unknown function $A(t)$ is $A(t)=\frac{f_{0} t}{\left.-2 \mathrm{i} \rho_{\mathrm{s}}+T k_{\text {perp }} / c\right)}$ so that the displacement $u(x, y=0, t)$ is

$$
u(x, y=0, t)=\frac{f_{0} t}{\left.-2 \mathrm{i} \rho_{\mathrm{s}}+T k_{\text {perp }} / c\right)} \mathrm{e}^{\mathrm{i} k_{\|} x-\mathrm{i} \omega t} .
$$

It is clear that the solutions with purely real frequencies increase linearly in amplitude to infinity, which is characteristic of a perfect resonance. The ratio $u(x, y=0, t) / f_{0}$ tends to infinity in this limit. This ratio also tends to infinity for the solutions with complex frequencies. However, the amplitude of the stimulus $f_{0} \mathrm{e}^{\mathrm{i} k_{\|} x-\mathrm{i} \omega t}$ goes exponentially to zero, so the amplitude of the solution effectively vanishes in long times. Having found the function $u(x, y, t)$ for $y=0$ in the time domain $t=0 \ldots \infty$ one can readily obtain its spatial $y$-dependence using its general form given in Eqs. (3) and (4). The envelope of this function with a complex frequency is given in Fig. 3.

\section{Conclusions}

Application of an external stimulus to the surface at the frequency (possible complex) corresponding to a surface resonance, be it a subsonic or supersonic leaky wave, results in a ratio of displacement of the surface layer to the amplitude of the stimulus tending to infinity for long times in a close analogy with the true surface wave. However, the amplitude of the displacement decreases with time for complex frequencies because the amplitude of the stimulus also tends to zero in an exponential manner. The maximum of the displacement in the time domain corresponds to the lifetime of the surface resonance. The spatial envelope of the leaky wave, entailed by a stimulus of frequency adjusted to the surface resonance, is directly given by the change of variable in Eq. (4) in the case of a bulk showing linear gapless dispersion relation. In the contrary case it is plausible to suppose that the overall

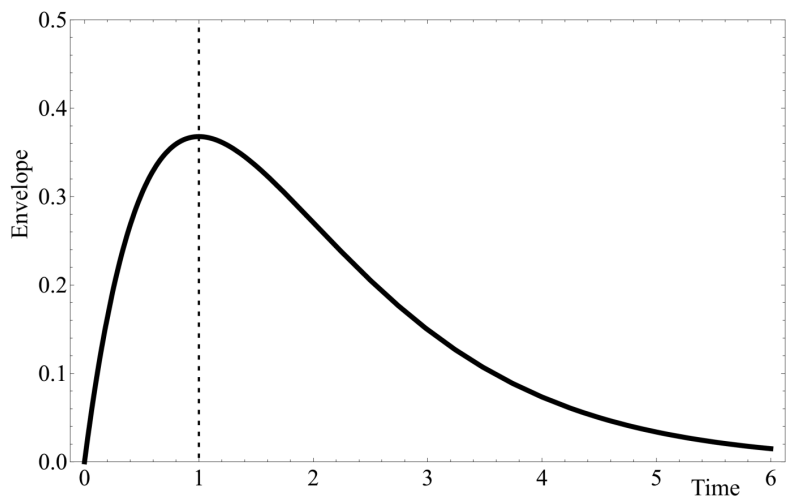

Fig. 3. Typical time-domain envelope of leaky wave produced by force showing damped-oscillating form adjusted to the frequency and lifetime of resonance. Spatial envelope can be deduced by inverting the sense of abscissa according to Eqs. (3) and (4).

shape is qualitatively similar but deformed due to the dispersion of the wave velocity. Subsonic surface waves have been recently found by the present authors [9] in a model of an elastic continuum with a hollowed cylinder cavity being a first approximation to the problem of surface waves in arteries. The physical manifestation of the subsonic leaky waves in an extra density of states in the close regions of the bulk bands might be important for physiological functions of arterial walls along with the bulk modes on the liquid and solid side of the blood wall interface [10].

\section{Acknowledgments}

This work is a part of the Thesis of D. Trzupek supported by the grant No N202 237337 of the Polish Ministry of Science and Higher Education.

\section{References}

[1] S.A. Gundersen, L. Wang, J. Lothe, Wave Motion 14, 129 (1991).

[2] A.G. Every, Phys. Rev. B 78, 174104 (2008).

[3] D. Twaróg, D. Trzupek, P. Zieliński, Acta Phys. Pol. A 115, 579 (2009).

[4] D. Twaróg, P. Zieliński, Phase Transit. 79, 577 (2006).

[5] D. Trzupek, P. Zieliński, Phys. Rev. Lett. 103, 075504 (2009).

[6] N.E. Glass, A.A. Maradudin, J. Appl. Phys. 54, 796 (1983).

[7] V.G. Mozhaev, M. Weihnacht, Ultrasonics 40, 927 (2002).

[8] P. Zieliński, Z. Łodziana, T. Srokowski, Prog. Surf. Sci. 59, 265 (1998).

[9] D. Trzupek, P. Zieliński, to be published.

[10] K. Jagielska, D. Trzupek, M. Lepers, A. Pelc, P. Zieliński, Phys. Rev. E 76, 066304 (2007). 